\title{
Low-medium resolution HLA-DQ2/DQ8 typing for coeliac disease predisposition analysis by colorimetric assay.
}

\author{
Hamdi Joda, Valerio Beni, Deidre Curnane, Ioanis Katakis, Noora Alakulppi, Jukka Partanen, \\ Kristina Lind, Linda Strombom and Ciara K. O’Sullivan
}

\section{Post Print}

N.B.: When citing this work, cite the original article.

The original publication is available at www.springerlink.com:

Hamdi Joda, Valerio Beni, Deidre Curnane, Ioanis Katakis, Noora Alakulppi, Jukka Partanen, Kristina Lind, Linda Strombom and Ciara K. O’Sullivan, Low-medium resolution HLADQ2/DQ8 typing for coeliac disease predisposition analysis by colorimetric assay., 2012, Analytical and Bioanalytical Chemistry, (403), 3, 807-819.

http://dx.doi.org/10.1007/s00216-012-5898-6

Copyright: Springer Verlag (Germany) http://www.springerlink.com/?MUD=MP

Postprint available at: Linköping University Electronic Press http://urn.kb.se/resolve?urn=urn:nbn:se:liu:diva-86352 


\title{
Low-Medium resolution HLA-DQ2/DQ8 typing for Coeliac Disease predisposition analysis by colorimetric assay.
}

\author{
Hamdi Joda ${ }^{1}$, Valerio Beni ${ }^{1,2}$, Deirdre Curnane ${ }^{1}$ Ioanis Katakis ${ }^{1}$, Noora Alakulppi ${ }^{3}$, Jukka Partanen ${ }^{3}$, \\ Kristina Lind ${ }^{4}$,Linda Strombom ${ }^{4}$, Ciara K. O'Sullivan ${ }^{1,5^{*}}$ \\ ${ }^{1}$ Departament d'Enginyeria Quimica, Universitat Rovira i Virgili, \\ Avinguda Països Catalans, 26, 43007 Tarragona, Spain \\ ${ }^{2}$ Current affiliation: Department of Physics, Chemistry and Biology (IFM), Linkoping University, S- \\ 58183, Linkoping, Sweden \\ ${ }^{3}$ Finnish Red Cross Blood Service, Kivihaantie 7, FIN-00310 HELSINKI, Finland \\ ${ }^{4}$ TATAA Biocenter AB, Odinsgatan 28, 41103 Göteborg, Sweden \\ ${ }^{5}$ Institucio Catalana de Recerca i Estudis Avançats, Passeig Lluis Companys 23, 08010, \\ Barcelona, Spain \\ * ciara.osullivan@urv.cat Fax: + 3497755 9621/67; Tel. + 34977558623
}

\begin{abstract}
Coeliac disease is an inflammation of the small intestine, occurring in genetically susceptible individuals triggered by the ingestion of gluten. Human Leukocyte Antigens (HLA) DQ2 and DQ8 gene have been identified as key genetic factors in coeliac disease as they are presented in almost $100 \%$ of the patients. These genes are encoded by the combination of certain alleles in the DQA and DQB region of chromosome 6. Specifically: DQA1*05:01 and DQB1*02:01 alleles for serologically defined leukocyte antigen DQ2 cis, DQA1*05:05 and DQB1*02:02 for DQ2 trans and DQA1*03:01 and DQB1*03:02 alleles for the DQ8. Specific identification of these alleles is a challenge due to the high number of alleles that have been identified so far: 46 in the DQA region and 160 in the DQB region (as of IMGT/HLA Database 10/2011 release).

In the reported work, the development of a multiplex colorimetric assay for the low to medium HLA typing of the DQ2 and DQ8 genes is presented. The optimisation of probe design and assay conditions, performed by both surface plasmon resonance and enzyme-linked oligonucleotide assay, are reported. Finally, the performances of the developed typing platform were validated by the analysis of real patient
\end{abstract}


samples. The HLA typing results gave excellent correlation when compared with those obtained using hospital based typing technologies.

Keywords: Coeliac disease, HLA typing, sequence specific oligonucleotide probes, SPR, ELONA

\section{Introduction:}

Coeliac disease (CD) is a chronic immune-mediated enteropathy of the small intestine occurring in genetically susceptible individuals; this is characterised by bowel mucosal inflammation, crypt hyperplasia, and villous atrophy[1,2]. CD is triggered by the ingestion of dietary gluten proteins that are present in wheat and to a lesser extent in barley and rye [3]. In the past CD was considered as a rare disorder of childhood, but more recent studies have shown that this is the most common disease in the western world affecting .even up to $1 \%$ of general population [4]. However, most CD cases remain undiagnosed due to the ambiguity of its clinical presentation or to its so called clinically silent forms [3]; this lack in diagnosis is associated with a decreased quality of life and numerous long-term complications such as osteoporosis, infertility, and small intestinal malignant lymphoma $[3,5]$

Currently $\mathrm{CD}$ diagnosis is based on clinical presentation and serological testing for anti-tissue transglutaminase (tTG) or endomysial (EMA) antibodies, confirmed by small intestinal biopsy, a rather invasive and expensive test [6,7]. Recently, the European Society for Paediatric Gastroenterology Hepatology and Nutrition has proposed a new diagnostic criteria based on the Delphi process, where this achieved using combination of HLA-typing and high titre levels of anti-tTG IgA antibodies, combined with detection of total IgA [8].

Several clinical studies have highlighted the relevance of HLA (Human Leukocyte Antigens) genes, located in chromosome 6 and CD susceptibility with ca. 95\% of patients have the CD-associated HLADQ2 gene and almost all the remaining patients express the HLA-DQ8 gene [2,5,9], with individuals negative to these genotypes being excluded from developing or having $\mathrm{CD}$ [10]

The HLA-DQ2 antigen is an heterodimer encoded by DQA1*05:01 and DQB1*02:01 alleles in its cis form or by DQA1*05:05 and DQB1*02:02 alleles in its trans form, whilst the HLA-DQ8 antigen is encoded by DQA1*03:01 and DQB1*03:02 alleles [1].

Classically, HLA typing was performed by serological methods but these methods are only suitable for low resolution typing and do not allow the identification of the different alleles [11,12]. The discovery of the polymerase chain reaction (PCR) considerably changed HLA typing leading to the development of PCR based typing methods including: sequence-specific primers (SSP)[13], sequence-specific oligonucleotide probe (SSOP) [14,15], single strand conformation polymorphism (SSCP) [16], 
restriction fragment-length polymorphism (RFLP) [17] and sequencing based typing [18]. In SSOP approaches (direct [19] or reverse [20] dot blot assays) the specificity of the assay is provided by the use of probes reverse complementary to allele specific regions of amplicons obtained using generic or group specific primers.

The growing importance of PCR based typing has resulted in the development of commercial kits for low resolution HLA typing of CD associate genes [21,22].

The enzyme-linked immunosorbent Assay (ELISA) [23], is a colorimetric affinity assay based on antigen antibody interaction, and is widely used in clinical practice as immuno diagnostic tool [24-27]. More recently "enzyme-linked oligonucleotide assay" (ELONA) [28], an affinity assay format analogue to the ELISA but based on oligonucleotide interaction has become increasingly popular. Godfroid et al. described a colorimetric microtitre plate hybridisation assay for the detection and identification of three types of human papillomaviruses (HPV) associated with cervical cancer [29]. Acero Sanchez et al. reported on a colorimetric sandwich ELONA for the rapid screening of 5 different genes related to breast cancer by detection of a single-stranded Multiplex ligation-dependent probe amplification (MLPA)-PCR product[30], Deng et al. developed ELONA methods for the detection of a single point mutation in M. tuberculosis genes [31] and Moribe et al. have detailed a reverse dot blot hybridisation assay for the rapid low resolution typing of class I (HLA-A, B and C) [20]. Allen et al. [32] and Kawai et al. [33] used a microtitre plate for typing of HLA class II DRB alleles and Legler et al. used the combination of multiplex polymerase chain reaction, ligase reactions and ELONA for the detection of human platelet antigen (HPA) systems HPA-1 through HPA-5 [34], highlighting the increased use of ELONA for DNA based diagnostics.

Surface Plasmon resonance (SPR) is an extremely powerful tool for monitoring of biomolecular interactions and has been extensively used for the detection of antigen-antibody [35], RNA- protein[36], DNA-protein[37] and DNA-DNA[38] interactions. Several reports on the use of SPR technique for DNA biosensing and its ability for single point mutation detection can be found in the literature [39-42].

In the work reported herein, the optimisation of the probe design and assay conditions, using both SPR and ELISA techniques, for the multiplex low-medium resolution HLA typing of the CD associated DQ2/DQ8 alleles and their implementation in an enzyme linked oligonucleotide assay is presented. SPR measurements were used, in first place, during probe design and assay condition optimisation; this choice was made because SPR provided and higher control in assay condition (temperature) and better capability for reliable screening of relatively large number of targets. The performances of the proposed sensing approach have been tested and validated by comparing them with those obtained at the Finnish 
Red Cross Blood Service (FRCBS) accredited Tissue typing laboratory using Luminex HLADQA1/DQB1 typing a standard technology for hospital laboratory based HLA typing.

\section{Material and Methods:}

All reagents used in this work were of analytical grade: Trizma hydrochloride, sulphuric acid, phosphate-buffered saline pH 7.4 with Tween 20 (PBS-T), Ethylenediaminetetraacetic acid disodium salt dehydrate (EDTA) and 3,3',5,5'-tetramethylbenzidine (TMB) liquid substrate system for ELISA were purchased from Sigma. $6 \mathrm{M}$ hydrochloric acid $(\mathrm{HCl})$, potassium dihydrogen phosphate $\left(\mathrm{KH}_{2} \mathrm{PO}_{4}\right)$, sodium chloride $(\mathrm{NaCl})$ and sodium dihydrogen phosphate $\left(\mathrm{NaH}_{2} \mathrm{PO}_{4}\right)$ were obtained from Scharlau. Sodium hydroxide $(\mathrm{NaOH})$ pellets were received from Panreac. Milli-Q water $(18 \mathrm{M} \Omega \mathrm{cm})$ was obtained using a Simplicity water purification system (Millipore, France). Reacti-Bind ${ }^{\mathrm{TM}}$ Maleimide Activated 96-Well Plates were supplied by Pierce. DNA probes, Horseradish peroxidise (HRP) modified reporting sequences (listed in Table 1), and synthetic analogues of relevant PCR amplicons were supplied by biomers.net (biomers.net GmbH, Germany). Single stranded DNA samples from patients were provided from TATAA Biocenter (Sweden). Further information regarding the amplicons and their synthetic analogues can be found in the supporting information (Table S1).

\section{Surface Plasmon Resonance (SPR)}

Surface Plasmon Resonance experiments were carried out using the Biacore 3000 (Uppsala, Sweden) using Biacore bare gold chips (Sensor Chip Au). Following cleaning the Au chip was docked in the instrument and flushed with PBS Tween buffer, at a flow rate of $15 \mu 1$ minute $^{-1}$, until stabilisation of the baseline was achieved. Probe immobilisation was performed by twice injecting $20 \mu \mathrm{l}$ of $1 \mathrm{M} \mathrm{KH}_{2} \mathrm{PO}_{4}$ containing $5 \mu \mathrm{M}$ of specific probe at a flow rate of $5 \mu 1$ minute $^{-1}$. Subsequently, a $1 \mathrm{mM}$ solution of mercaptohexanol $(\mathrm{MCH})$ was injected at a flow rate of $5 \mu 1$ minute $^{-1}$ for 4 minutes to remove/displace weakly bound probes and to reduce non-specific adsorption of the DNA targets during the hybridisation. Specific and non-specific synthetic alleles were diluted to $250 \mathrm{nM}$ in hybridisation buffer (10 mM Tris buffer containing $1 \mathrm{M} \mathrm{NaCl} \mathrm{pH} \mathrm{7.4)} \mathrm{and} \mathrm{injected} \mathrm{for} 5$ minutes at a flow rate of $5 \mu 1$ minute $^{-1}$. The surface of the chip was regenerated via a 1 minute wash with $15 \mathrm{mM} \mathrm{NaOH}$ to leave the chip surface still functionalised with the immobilised probe.

\section{ELONA hybridisation assay}


Thiol functionalised probes were immobilised on maleimide activated microtitre plates following the manufacturer's instructions. Briefly, $100 \mu \mathrm{l}$ of $500 \mathrm{nM}$ of the specific probe in binding buffer $(0.1 \mathrm{M}$ sodium phosphate, $0.15 \mathrm{M}$ sodium chloride, $10 \mathrm{mM}$ EDTA; $\mathrm{pH}$ 7.2) were added to each well of the microtitre plate and incubated for 2 hours at $37^{\circ} \mathrm{C}$. Subsequently, $100 \mu \mathrm{l}$ of mercaptohexanol $(1 \mathrm{mM})$ were added to each well (incubation 1 hour at $37^{\circ} \mathrm{C}$ ) in order to inactivate excess maleimide groups. Hybridisation was performed by addition of $100 \mu \mathrm{l}$ of a $20 \mathrm{nM}$ of the synthetic alleles solutions in hybridisation buffer (10 mM Tris buffer containing $1 \mathrm{M} \mathrm{NaCl} \mathrm{pH} \mathrm{7.4)} \mathrm{or} \mathrm{in} \mathrm{case} \mathrm{of} \mathrm{real} \mathrm{patients} \mathrm{sample}$ analysis, amplicons of the PCR product (diluted 1 to 10 in hybridisation buffer). Hybridisation with the target DNA was allowed to proceed for a pre-defined time at a controlled temperature. Subsequently 10 $\mathrm{nM}$ of the reporter probe in hybridisation buffer was added and left to hybridise for a given time at a controlled temperature. Between each step the plates were washed three times with PBS-Tween using an automated microplate washer.

To detect the presence of captured DNA, TMB liquid substrate was added (100 $\mu$ l) to each well and incubated for 5 minutes. Following incubation and colour development the reaction was stopped by addition of $1 \mathrm{M} \mathrm{H}_{2} \mathrm{SO}_{4}$ and the absorbance measured at $450 \mathrm{~nm}$ using a microplate reader (EMax, Molecular Devices, BioNova Científica).

\section{Multiplex PCR amplification and ssDNA generation:}

Blood samples were collected from CD affected and/or suspected patients at Kuopio University Hospital (Finland). DNA isolation was performed using the Qiagen FlexiGene DNA kit, and HLA typing was performed in the accredited Finnish Red Cross Blood Service (FRCBS) Tissue typing laboratory. Typing was carried out using two different approaches: Luminex HLA-DQA1/DQB1 typing and confirmed by Olerup DQA1 or DQB1 SSP typing kits.

Multiplex PCR amplification was performed using iQ Multiplex Powermix from Bio-Rad in the presence of $20 \mathrm{ng}$ of DNA template. Amplification was performed in $200 \mu \mathrm{L}$ tube $(50 \mu 1$ reaction) format using an RotorGene 6000 thermocycler according to the following protocol: i) initial denaturation at 95 ${ }^{\circ} \mathrm{C}$ for 10 minutes and ii) 40 cycles $\left(15\right.$ seconds at $95{ }^{\circ} \mathrm{C}$ and 60 seconds at $\left.60{ }^{\circ} \mathrm{C}\right)$.

For the multiplex amplification three primer pairs, specifically designed for low to medium resolution DQ8-DQ2 typing, were used: DQA exon 2 (200 nM), DQA exon 3 (200 nM), DQB exon2 (200 nM) and DQB exon3 (100 nM). All the reverse primers used in the reported work were phosphorylated at their 5', end in order to allow enzymatic generation of single stranded DNA via Exonuclease digestion.

\section{ssDNA Generation}


In order to facilitate detection of the PCR product, generation of ssDNA is required. In the reported work generation of ssDNA was performed by means of Lambda exonuclease (Fermentas GmbH) mediated hydrolysis. Lambda exonuclease is a highly processive 5' to 3' exodeoxyribonuclease, which is able to selectively digest the phosphorylated strand of dsDNA but it is characterised by a reduced activity on ssDNA and non-phosphorylated DNA [43,44]. Single stranded DNA was generated by incubating the $50 \mu \mathrm{l}$ PCR product, in the presence of $2 \mu 1(20 \mathrm{U})$ of the exonuclease for 30 minutes at 37 ${ }^{\circ} \mathrm{C}$. Following the digestion the solution was heated to $80{ }^{\circ} \mathrm{C}$ for 10 minutes in order to inactivate the enzyme. Efficiency of ssDNA generation was tested by melting curve analysis as reported in the supporting information (Figure 1S).

\section{Results and discussions}

In the reported work the development of an ELONA assay, based on a Sequence Specific Oligonucleotide Probes (SSOP) hybridisation format, for the low to medium resolution typing of CD associated HLA DQ2-DQ8 antigens is presented. HLA-DQ2 is encoded by DQA1*05:01/DQB1*02:01 alleles in its cis format and by DQA1*05:05/DQB $1 * 02: 02$ alleles in the trans format. HLA-DQ8 is encoded by DQA1*03:01/ DQB1*03:02 alleles (Scheme 1).

In the reported work, low resolution assays are defined as the detection of allele groups without discrimination of specific alleles; for example probe DQB02gen enables the detection of the whole DQB1*02 group but not the discrimination between members of the group. Medium resolution assays are defined as the detection of multiple alleles within the same group but not the identification of a single allele; for example probe DQA05 enables the detection of both DQA1*05:01 and DQA1*05:05 alleles but it does not allow their discrimination. High resolution typing is defined as when the probe used is able to specifically identify a specific allele as in the case of the DQA0201 probe. The primary identification of the probes and of potentially interfering alleles was performed with the help of the IMGT/HLA database [45], the handbook of the $13^{\text {th }}$ International Histocompatibility Working Group (IHWG) [46] and multiple sequence alignment software (Genedoc) [47]. The possible interfering alleles were chosen according to similarity and mismatches at the probe region. Alleles sharing the same sequence at the probe location were represented by one allele to be used in the assay. Finally, generic regions in the target alleles were identified for the design of different reporting sequences. Sequences of the different probes and reporting sequences, together with description of the synthetic targets/interfering alleles (sequences at probe region) are reported in Table 1. 


\section{Probes and interference alleles selection:}

$D Q 2$

$D Q A 1 * 05: 01 / 05: 05$

DQA05 probe was designed to recognise a sequence in the exon 3 DQA1 locus common to both DQA1*05:01 and DQA1*05:05 alleles, obtaining in this case a medium resolution typing of the DQA1*05 alleles of interest for CD. This probe was also able to detect DQA1*05:08, DQA1*05:09, and DQA1*05:10 (Table 1) alleles that are not associated to CD but that have a very low prevalence [48]. The specificity of the probe was tested against 3 potentially interfering alleles, DQA1*05:03, DQA1*02:01 and DQA1*03:02 presenting 1, 2 and 3 base mismatches, respectively. These alleles, described in Table S1 were representative for all the known alleles in DQA1 locus at the probe region.

\section{$D Q B 1 * 02: 01 / 02: 02$}

DQB1 locus is more polymorphic than the DQA1, thus complicating the genotyping. A combination of two probes was required in order to perform the low resolution typing of DQB1*02:01 and DQB1*02:02 alleles, where the probes were designed to recognise two different regions of exon 2 of the DQB1 locus. The first probe, namely DQB02A, was designed to recognise the region between codons 52 and 58 (Table 1), which is a region shared by all the alleles in the DQB $1 * 02$ group with the exception of DQB1*02:03. For this probe a total of 5 potentially interfering allele groups with 1, 2, 3, 4 and 5 base mismatches, respectively (Table S1) were identified, probes that can detect some rare DQB1 alleles (DQB1*02:04, DQB1*02:05 and DQB1*02:06) that are not related to CD (Table S1) but that have very low prevalence $(<1 \%$ of the human population) [48] .

Probe DQB02A is expected to have high cross hybridisation with the DQB1*03:02 allele and in order to improve the specificity of the assay the introduction of an additional probe was required. This further probe, DQB02gen, located one codon to the right side of the DQB02A probe, is able to recognise all of the DQB1*02 alleles group and has 2-3 nucleotide mismatches as compared to other DQB1 alleles. Again 3 potentially interfering allele groups, represented by the alleles DQB1*03:02, DQB1*04:01 and DQB1*05:01, were identified and used to test the specificity of the probe (Table 1S).

\section{$D Q A 1 * 02: 01$}

In the case of this allele, the probe was designed in the exon 2 between codon 51 and 58, in a region with multiple nucleotides substitution/deletion between the perfect match (DQA1*02:01) and other 
DQA alleles. To test discrimination ability of the DQA0201 probe, 3 alleles: DQA1*01:01, DQA1*05:01 and DQA1*03:01, which represent the all the possible interfering alleles in the DQA Exon 2 probe region, were used (Table 1S).

\section{DQ8}

$D Q B 1 * 03: 02$

DQB $1 * 03$ allele is characterised by a large number of subgroups, making high resolution genotyping of alleles belonging to this group quite difficult. In order to achieve good resolution for the detection of the DQB1*03:02 allele, two probes (Table 1) were designed. Each probe was designed to exclude several non specific alleles, with the combination of the information recorded from these two probes facilitating identification of the DQB1*03:02 allele. The first probe (DQB0302A) is located between codon 45-49 of exon 2 of the DQB1 locus. This probe detects both the target allele as well as several alleles not related to $\mathrm{CD}$ (Table 1 ) including the DQB $1 * 04$ group. In order to improve typing specificity the second probe (DQB0302B), located 5 codons to the 3' direction, was designed.

The combination of the two DQB03 probes allowed specific detection of the DQB1*03:02 allele. Again some rare alleles were also detected; these alleles, DQB1*03:05, DQB1*03:08, DQB1*03:11 and DQB1*03:18, have a very low prevalence $(<1 \%)$ in the Caucasoid population [48], except for the DQB1*03:05 which is present in some population originally from Sardinia (Italy) $[48,49]$. Potentially interfering alleles (Table 1S) were designed to allow evaluation of the specificity and selectivity of the two probes: 3 alleles were identified for the DQB0302A probe and 5 for the DQB0302B probe.

\section{$D Q A 1 * 03$}

A probe for low resolution genotyping of the DQA $1 * 03$ allele was designed in a region partially overlapping the DQA1*02:01 probe. The same potentially interfering alleles used for the DQA1*02:01 allele with the in addition of the DQA $1 * 02: 01$ allele were used to test specificity of this probe.

\section{Surface Plasmon Resonance (SPR)}

SPR experiments were carried out to evaluate in high throughput format, the specificity of the designed probes. As expected this was a function of the number of mismatches with higher nonspecific response associated with the alleles characterised by a single base mismatch.

As well established [50], increasing the assay temperature allows better discrimination between fully complementary and mismatched targets and thus the effect of the hybridisation temperature (ranging from $20^{\circ} \mathrm{C}$ to $40{ }^{\circ} \mathrm{C}$ ) on capture efficiency and probe specificity was tested. Figure 1 shows the changes 
in SPR responses, as a function of hybridisation temperature, obtained at the different probes for the hybridisation of the fully complementary targets. For all the SPR experiments response was obtained for the dissociation part of the sensongrams. Example of a typical set of temperature experiments can be found in the supporting information (Figure 2S). Interestingly, two different trends in the probe behaviour were observed. In the case of the DQA1 probes (DQA0201, DQA03 and DQA05) the SPR signal was higher with increasing temperature until a maximum was reached, between 25 and $30{ }^{\circ} \mathrm{C}$. Further increases in hybridisation temperature resulted in a significant drop in SPR response, especially for the DQA0201 and DQA03 probes.

In the case of the DQB1 probes (except DQB0302A probe) hybridisation responses increased with assay temperature. SPR signal for DQB0302A probe is almost constant throughout the different temperatures investigated.

In order to interpret the results obtained, the probe secondary structure was evaluated, as this is known to influence hybridisation [51-53]. A prediction of the presence of secondary structures was performed by calculating the melting temperature $(T m)$ and free energy $(\Delta \mathrm{G})$ of the self-complementary secondary structure of the different probes using Mfold Web Server [54], using a salt $\left(\mathrm{Na}^{+}\right)$concentration of $1 \mathrm{M}$ (those used in the hybridisation assay). From the simulation the following thermodynamic parameters for the different probes were obtained: DQA0201; $T m=11^{\circ} \mathrm{C}, \Delta G=0.59 \mathrm{kcal} / \mathrm{mol}$, DQA03; $T m=23.1$ ${ }^{\circ} \mathrm{C}, \Delta \mathrm{G}=0.15 \mathrm{kcal} / \mathrm{mol}$, DQA05; $T m=25.4{ }^{\circ} \mathrm{C}, \Delta \mathrm{G}=-0.02 \mathrm{kcal} / \mathrm{mol} \mathrm{DQB} 02 \mathrm{~A} ; \mathrm{Tm}=56.7{ }^{\circ} \mathrm{C}, \Delta \mathrm{G}=-$ $3.06 \mathrm{kcal} / \mathrm{mol}, \mathrm{DQB} 02 \mathrm{gen} ; \mathrm{Tm}=52.7{ }^{\circ} \mathrm{C}, \Delta \mathrm{G}=-2.62 \mathrm{kcal} / \mathrm{mol}, \mathrm{DQB} 0302 \mathrm{~B} ; \mathrm{Tm}=50.4{ }^{\circ} \mathrm{C}, \Delta \mathrm{G}=-1.96$ $\mathrm{kcal} / \mathrm{mol}$. No secondary structure was predicted for DQB0302A probe.

In the case of the DQA1 probes no significant influence from the possible secondary structures of the probes can be expected as these are characterised by the low melting temperatures $\left(11-25.4{ }^{\circ} \mathrm{C}\right)$; consequently hybridisation response can be expected to be dominated by the stability (melting temperature) of the probe/target duplex (Table 1). In the case of the DQB1 probes the possible secondary structures were predicted to be very stable with melting temperature above $50{ }^{\circ} \mathrm{C}$ probably due to their high G-C content. In this case, the possible secondary structures are expected to compete with the hybridisation of the target DNA reducing the efficiency of the recognition event. When the hybridisation temperature was raised, considerable increases in SPR response were recorded, consistent with the fact that high temperature results in destabilisation of the secondary structures facilitating the hybridisation between the target and the probe. 
Additionally, different probes were observed to have highly variable hybridisation efficiencies at low temperature, SPR signal for the most of probes are converged at higher temperature to cross each other in temperature between 35 and $40{ }^{\circ} \mathrm{C}$ (Figure 1). In Table 2 a summary of the normalised responses obtained for the most relevant interfering sequences is reported. For the majority of the probes, an increase in specificity was recorded with increasing temperature. This improvement was more relevant in the case of the DQA1 and DQB02gen probes where the responses recorded for the interfering alleles were considerably reduced from ca $20 \%-40 \%$ (at $20{ }^{\circ} \mathrm{C}$ ) to less than $10 \%$ at $40{ }^{\circ} \mathrm{C}$. In the case of the DQB1 probes the improvement in specificity was much lower: from ca. $70 \%-85 \%$ to ca. $40 \%-70 \%$. This reduced improvement could still be associated with the high G-C content of these probes that can provide considerable stability also for the mismatched duplex even at high temperature. A further element that could play a role in the specificity of the assay is the position [55] and nature [56,57] of the mismatched base. Kelley et al. demonstrated that sequences with mismatches far from the electrode surface, as in the case of DQB1*02:03, have higher stability than those with mismatches located closer to the surface, as in the case of DQA1*05:03 [55]. Furthermore, Allawi et al. showed that different base pairs possess different thermodynamic stabilities [56,57] with the C-T pair mismatch (as in the case of DQA1*05:03) being less stable than the G-A mismatch as is present in the DQB1*02:03 allele.

The Surface Plasmon Resonance studies revealed the importance of temperature in the optimisation of the selectivity and sensitivity of the proposed probes. As seen in Table 2, it is clear that hybridisation assay should be performed at elevated temperatures, and thus it was decided to perform the ELONAs at $37{ }^{\circ} \mathrm{C}$ despite the fact that at this temperature the responses at the DQA0201 and DQA03 probes are considerably suppressed.

\section{Enzyme Linked OligoNucleotide Assay (ELONA)}

\section{Probes specificity evaluation:}

Following the preliminary SPR screening a series of ELONAs were performed to confirm the specificity of the different probes in the measurement condition. In this set of experiments probes immobilised on maleimide activated plates were hybridised with the designed potentially interfering sequences (described in Table 1S).

\section{$D Q 2$}

$D Q A 1 * 05: 01 / 05: 05$

As can be seen from Figure 2A the proposed DQA05 probe presented an excellent specificity for the DQA1*05:01 allele over the possible interfering alleles with only the DQA1*05:03 allele with a single 
base mismatch (G to $\mathrm{T}$ ) giving a relatively considerable interfering signal (lower than $25 \%$ of the fully complementary allele (DQA $1 * 0501)$.

\section{$D Q B 1 * 02: 01 / 02: 02$}

In the case of DQB02A probe, a good specificity over the DQB1*03:01, DQB1*04:01 and DQB1*05:01 alleles was achieved (Figure 2B). As expected the DQB1*02:03 and DQB1*03:02 alleles gave the highest interference with response associated to them being respectively the $57 \%$ and $44 \%$ of those obtained for the fully complementary target. In Figure $2 \mathrm{C}$ the results obtained using the DQB02gen probe are presented. The use of this probe allowed a good discrimination of the DQB $1 * 0302$ allele (response lower than $12 \%$ of the specific signal), confirming the possibility of detecting the DQB1*02:01 and DQB1*02:02 alleles with a good specificity using the combination of the two DQB probes.

\section{$D Q A 1 * 02: 01$}

Probe DQA1*0201 allows high resolution typing for the DQA1*02:01 allele as this is the only allele in the DQA $1 * 02$ family. As can be observed in Figure 2D, this probe was demonstrated to have a very high specificity for the fully complementary allele, due to the multiple substitutions and deletions (3-bp deletion at codon 56) between the perfect match allele and the possible interfering alleles (DQA1*01:01, DQA1*03:01 and DQA1*05:01).

\section{DQ8}

\section{$D Q B 1 * 03: 02$}

In Figure $2 \mathrm{E}$ and Figure $2 \mathrm{~F}$ the results of the cross hybridisation studies for the DQB $1 * 0302$ probes (DQB0302A and DQB0302B) are presented. Probe DQB0302A allowed good specificity for the DQB1*03:02 against the interfering targets DQB1*02:01 and DQB1*03:01 as the potentially interfering sequences have between 2 and 3 nucleotide mismatches. However, a high non-specific hybridisation signal (ca. 60\% of the specific response) was obtained for the DQB1*05:01 allele, characterised by one base mismatch located at the terminal end.

In order to reduce the possibility of false positives a second probe (DQB0302B) was introduced and this second probe provided a good specificity for the DQB $1 * 03: 02$ allele with a lower than $35 \%$ response for the DQB1*03:01 allele (major interfering sequence). This probe allowed the discrimination of the DQB1*03:02 from the DQB1*04:01 and DQB05:01 alleles, the major potentially interfering alleles for the DQB03A probe. 


\section{$D Q A 1 * 03$}

The selectivity of the DQA03 probe was tested in the presence of specific and non-specific alleles and the probe was demonstrated to be very specific with very low non-specific hybridisation, lower than $3 \%$ of the specific response, (Figure $2 \mathrm{G}$ ).

\section{Hybridisation time/temperature}

Optimisation of the timing of the different steps (target hybridisation and reporting sequence hybridisation) is extremely important in affinity assays influencing as these factors affect both the response intensity and the specificity [30]. An optimisation of assay time was performed for two probes (DQA03 and DQB02A), one for each of the group identified during the SPR evaluation, at two different temperatures $\left(22{ }^{\circ} \mathrm{C}\right.$ and $\left.37{ }^{\circ} \mathrm{C}\right)$. In this experiment, the capture of $20 \mathrm{nM}$ of the fully complementary alleles and $10 \mathrm{nM}$ of the reporting element was used and hybridisation times between 5 and 60 minutes were evaluated.

To optimise the target hybridisation time, the capture of the reporting element was performed for 60 minutes, and optimisation of the hybridisation with the labelled reporter probe was carried out using an optimised 5 minutes target hybridisation time. In Figure $3 \mathrm{~A}$ the results obtained for the target hybridisation time at the two temperatures for the DQA03 probe are presented. For this probe no significant differences were recorded when the target hybridisation was performed at $22{ }^{\circ} \mathrm{C}$ or $37{ }^{\circ} \mathrm{C}$ and the same set of experiments indicated that 5 minutes is adequate for target hybridization, consistent with results reported by Acero et al, [30]. Whilst a different behaviour was recorded in the case of the DQB02A probe, where the use of higher temperature $\left(37^{\circ} \mathrm{C}\right)$ significantly influenced the hybridisation with the target (Figure 3B). These behaviours were consistent with those found with SPR measurements and discussed extensively above.

The study of the hybridisation time of the HRP labelled reporter probe demonstrated that in the case of the DQA03 probe a significant increase in response was recorded when this step was performed at $37^{\circ} \mathrm{C}$ (Figure 3C), whilst no significant influence of the temperature was observed in the case of the DQB02A probe (Figure 3D).

As it can be observed from Figure 3, 5 minutes for target hybridisation, 20 minutes for hybridisation with the HRP-labelled secondary probe and $37{ }^{\circ} \mathrm{C}$ can be considered a good compromise between assay time and assay performances. As it can be seen from Figure 3A and Figure 3B at $37{ }^{\circ} \mathrm{C}$ hybridisation between the targets and the on-plate immobilised probes was already very efficient even after only 5 minutes. In the case of the capture of the secondary probe interpretation of the results was more 
uncertain; for both the probes investigated (DQA03 and DQB02A) responses were increasing with the time but at it can be seen especially in the case of probe DQA03 (Figure 3C) this increase was not so relevant for times longer than 20 minutes. On the other end in the case of the DQB02A probe responses were increasing significantly with the time (Figure 3D). The fact that for 20 minutes of capture time similar responses were recorded for the two probes suggested us that this was an adequate time for this step of the assay.

In an attempt to further reduce the assay time the possibility of performing the assay in a single step format, by pre-incubating the target oligonucleotides and the reporting sequence, was evaluated. As it can be seen in the supporting information this approach (Figure 3S) did not improved the assay performances.

\section{Reporting label cross-reactivity.}

In order to perform multiplex detection of the alleles required for the low to medium resolution typing of the DQ2-DQ8 genes, use of different reporter probes, one for DQA exon2, one for DQA exon 3 and one DQB exon2, is required (Table 1). Prior to performing multiplex HLA typing the possible interaction between the different reporting elements was investigated, by performing ELONA experiments using a solution containing the specific reporter probes or a mixture of the three reporter elements at equivalent concentrations of $10 \mathrm{nM}$. The mixture of the three different reporter probes was prepared and left to incubate for 10 minutes at $37^{\circ} \mathrm{C}$ previous to use, in order to allow the possible interaction between the reporter probes to take place.

As can be seen in Figure 4 (results obtained for the DQA03 probe) no significant differences were recorded when the reporter probes were added as a single component or in a mixture.

\section{Detection of real samples:}

Following optimisation of the design of the probes and assay conditions with synthetic analogues of the different PCR products, an analysis of PCR product from real patients sample was carried out. In this evaluation, 4 previously typed patients samples and 1 cell line (LZL) from European Collection of Cell Cultures were used. The different samples were HLA typed in the accredited FRCBS Tissue typing laboratory using different methods: Luminex One Lambda Labtype ${ }^{\circledR}$ SSO HLA Class II DQA1/DQB1 Typing Test, and Olerup DQA1or DQB1 SSP typing kits. Multiplex PCR amplification and ssDNA generation was performed in TATAA Biocenter.

The prepared samples were frozen and stored to $-20{ }^{\circ} \mathrm{C}$. Sample analysis was performed, with 1 in 10 dilution in hybridisation buffer followed by hybridisation with the 7 probes (three repetitions for each probe) and performing the detection according to the optimised protocol. In Figure 5 the normalised 
results (ration between specific response and control) are presented. The results of ELONA typing, together with those obtained using reference typing techniques (Luminex HLA-DQA1/DQB1 typing and confirmed by Olerup DQA1 or DQB1 SSP typing kits) are summarised in Table 3.

In this table the scores to the responses at the different probes were assigned according to the following criteria:

When the ratio between the response for the sample for each probe and the control experiment was lower than 5 the sample was considered negative for that probe; if the ratio was higher than 15 the sample was considered positive. All the value in between were judged accordingly to the results reported in Figure 2. A detailed description on how the samples' typing was obtained is presented in the supporting information.

The predicted HLA typing for the different samples was consistent with those obtained using standard HLA typing techniques (Table 3). Clearly the proposed ELONA approach allowed HLA typing of the sample with a very good level of reproducibility and specificity.

\section{Conclusions}

In the proposed work, a set of probes for the low to medium resolution genotyping of $\mathrm{CD}$ associated genes has been designed and tested. The complexity of the HLA system did not allow the use of a single probe for each targeted allele making necessary the use of combination of probes to obtain the desired information. The use of the combination of several probes allowed specific identification of a specific alleles or of a restricted group of them (as in the case of the DQB $1 * 02$ alleles).

SPR and ELONA experiments were used to identify the optimal conditions, in terms of assay temperature and timing of the different steps, to perform the proposed assay.

SPR results indicated that the various probes had quite different behaviour in relation with the hybridisation temperature; this made necessary to find a suitable compromise. In the reported work assay was performed at $37{ }^{\circ} \mathrm{C}$ that allowed optimal detection of the different DQ2/DQ8 alleles. Optimisation of the timing of the different steps involved in the detection showed that detection was possible using 5 minutes hybridisation time and 20 minutes label capture time limiting the total assay to less than 30 minutes.

Finally the assay was also shown to be suitable for the HLA typing of real samples confirming the applicability of the proposed assay format for accurate $(\% \mathrm{RSD}<15 \%) \mathrm{CD}$ predisposition analysis.

\section{Acknowledgements}


This work was carried out with the financial support from the Commission of the European Communities, specific RDT program "Coeliac Disease Management Monitoring and Diagnosis using Biosensors and an Integrated Chip System, CD-MEDICs, FP7-2007-ICT-1-216031”. V. Beni acknowledges the European Union's Seventh Framework Program (FP7/2007-2013) under grant agreement no. PIGF-GA-2008-220928 for financial support. H. Joda thanks Universitst Rovira i Virgili for the doctoral scholarship. We thank Dr. Markku Heikkinen, MD, PhD (Kuopio University Hospital, Kuopio, Finland) for blood samples of Finnish coeliac disease patients and Dr. Taina Jaatinen, PhD, Dr. Katri Haimila, PhD and Juha Peräsaari, MS (Finnish Red Cross Blood Service, Helsinki, Finland) for HLA tissue typing expertise.

\section{References:}




\section{TABLES AND FIGURES}

Scheme 1: Schematic description of the different possible allele combinations encoding the DQ2 and DQ8 genes.

Table 1: Sequences of the probes and reporting elements.

Table 2: SPR evaluation of the effect of temperature on the sensitivity and selectivity of all SSO probes used in this report. Temperature was investigated in the range of $20-40{ }^{\circ} \mathrm{C}$. For each probe, $\%$ values were calculated as a ratio between RU responses obtained for the interference allele under evaluation and those obtained for the fully complementary allele.

Table 3: Summary of the HLA typing of the sample using the proposed ELONA assay and its comparison with the typing obtained with the reference approaches used in this work: Luminex HLADQA1/DQB1 typing and confirmed by Olerup DQA1 or DQB1 SSP typing kits.

Figure 1: Plot of the RU variation resulting from the hybridisation of the fully complementary targets for the different probes as a function of the hybridisation temperature. Each value is the result of three sequential injection/regeneration cycles.

Figure 2: Evaluation of the specificity of the different probes designed for the DQ2-DQ8 typing: DQA05 (A), DQB02A (B) DQB02gen (C), DQA0201 (D), DQB0302A (E), DQB0302B (F) and DQA03 (G). Hybridisation was performed in the presence of $20 \mathrm{nM}$ of the targeted DNA for 5 minutes at $37^{\circ} \mathrm{C}$ and capture of reporting sequence was performed from a $10 \mathrm{nM}$ solution for 20 minutes at $37{ }^{\circ} \mathrm{C}$. The absorbance values were normalised to the reading of the fully complementary allele. 
Figure 3: Evaluation of the target hybridisation time (A and $\mathrm{B}$ ) and of the capture time of the reporting elements (C and D) for the DQA03 (A and C) and DQB02A (B and D) probes. ELONA assays were performed at 37 and $22^{\circ} \mathrm{C}$ and using $20 \mathrm{nM}$ of target DNA and $10 \mathrm{nM}$ of reporting element.

Figure 4: ELONA evaluation of the possible cross reactivity between the different reporting elements required for the multiplex detection according to the proposed detection platform. Study performed by using DQA03 probe and using $20 \mathrm{nM}$ of target DNA and $10 \mathrm{nM}$ of reporting element.

Figure 5: Colorimetric multiplex analysis of 4 real samples and cell line sample LZL. Hybridisation was carried out using PCR samples diluted 1:10 for 5 minutes. For detection, a mixture of reporter probes (10 nM each) was incubated for 20 minutes at $37{ }^{\circ} \mathrm{C}$. Data presented as ration between the specific response and the control response. 


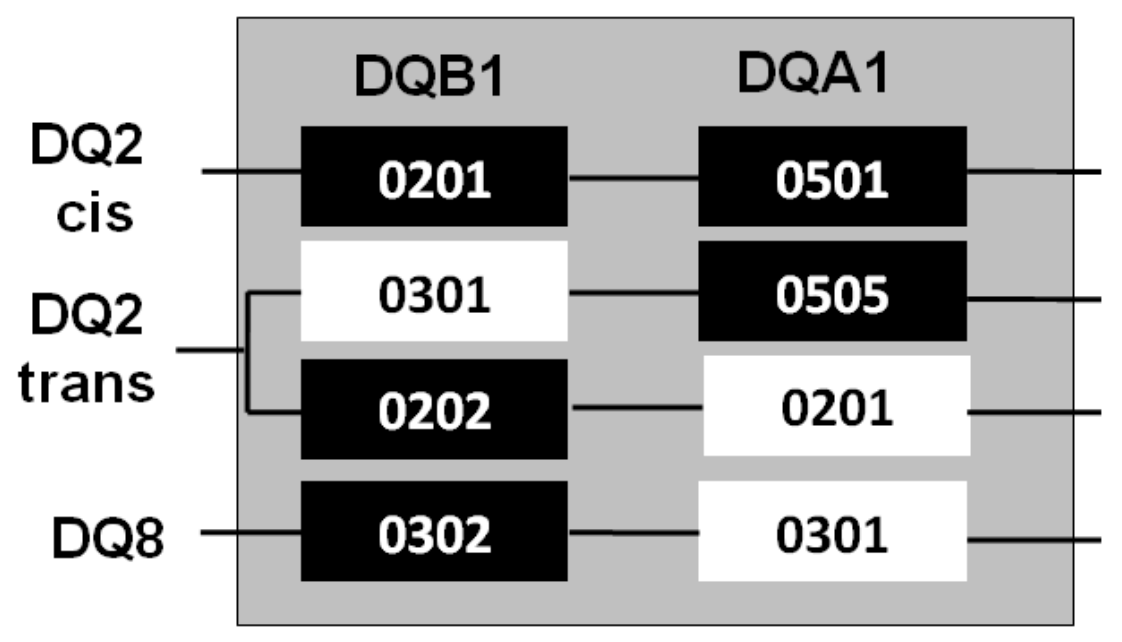

Scheme 1 


\begin{tabular}{|c|c|c|c|c|c|}
\hline \multicolumn{6}{|c|}{ Probes } \\
\hline Probe name & Probe sequence & $\begin{array}{l}\text { IMGT/HLA } \\
\text { Matched } \\
\text { alleles }\end{array}$ & codon & $\operatorname{Tm}{ }^{\circ} \mathrm{C}$ & GC\% \\
\hline DQA0201 & 5' SH-C6-CAAATCTAAGTCTGTGGA 3' & DQA $1 * 02: 01$ & $58 \_1-51 \_2$ & 36 & 38.9 \\
\hline$D Q A 03$ & $\begin{array}{l}\text { 5' SH-C6- CTTCTAAATCTGCGGAACA } \\
\text { 3' }\end{array}$ & $\begin{array}{l}\text { DQA1*03:01, } \\
03: 02,03: 03\end{array}$ & $56 \_2-50 \_2$ & 45 & 42.1 \\
\hline$D Q A 05$ & 5' TAACTCTCCTCAGCAGA-C3-SH 3' & $\begin{array}{c}\text { DQA1 } * 05: 01, \\
05: 05,05: 08, \\
05: 09\end{array}$ & $164 \_2-159$ & 36 & 47.1 \\
\hline$D Q B 02 A$ & 5' CGGCAGGCAGCCCCAGCA-C3-SH 3' & $\begin{array}{c}\text { DQB } 1 * 02: 01, \\
02: 02,02: 04, \\
02: 05\end{array}$ & $58 \_1-52 \_2$ & 65 & 77.8 \\
\hline DQB02gen & 5' AGGCAGCCCCAGCAGCG-C3-SH 3' & $\begin{array}{l}\text { DQB1 } 1 * 02: 01, \\
02: 02,02: 03, \\
02: 04,02: 05\end{array}$ & $56-51 \_2$ & 59 & 76.5 \\
\hline$D Q B 0302 A$ & 5' CGCCCGATACACCCC-C3-SH 3' & $\begin{array}{c}\text { DQB } 1 * 03: 02, \\
03: 03,03: 05, \\
\text { 03:06, 03:08, } \\
\text { 03:10-12, 03:15, } \\
\text { 03:17-18, 03:20, } \\
\text { 04, 05:02:02, } \\
\text { 05:03, 06:01, } \\
06: 19\end{array}$ & $49 \_3-45 \_1$ & 52 & 73.3 \\
\hline$D Q B 0302 B$ & 5' TCGGCGGCAGGCGGC-C3-SH 3' & $\begin{array}{l}\text { DQB1 } * 03: 02, \\
03: 04,03: 05, \\
03: 07,03: 08, \\
03: 11,03: 14, \\
03: 18,06: 29\end{array}$ & 59_2 - 54_3 & 56 & 86.7 \\
\hline \multicolumn{6}{|c|}{ Reporting probes } \\
\hline $\begin{array}{l}\text { Reporting } \\
\text { element name }\end{array}$ & Reporting element sequence & Target exon & \multicolumn{3}{|c|}{ Codon } \\
\hline DQAex2 & 5'GACAGTCTCCTTCCTCTC - HRP '3 & DQA Exon 2 & \multicolumn{3}{|c|}{ 45_3 - 40_1 } \\
\hline DQAex3 & 5' HRP-CCCCAGTGTTTCAGAAGA 3' & DQA Exon 3 & \multicolumn{3}{|c|}{ 182_1 - 176_3 } \\
\hline$D Q B e x 2$ & 5' HRP-CTGGTAGTTGTGTCTGCA 3' & DQB Exon 2 & \multicolumn{3}{|c|}{$84 \_3-79 \_1$} \\
\hline
\end{tabular}

Table 1 


\begin{tabular}{|c|c|c|c|c|c|}
\hline \multirow[t]{2}{*}{ Allele } & \multicolumn{5}{|c|}{ Normalised SPR response } \\
\hline & $20^{\circ} \mathrm{C}$ & $25^{\circ} \mathrm{C}$ & $30^{\circ} \mathrm{C}$ & $35^{\circ} \mathrm{C}$ & $40^{\circ} \mathrm{C}$ \\
\hline \multicolumn{6}{|c|}{ DQA05 Probe } \\
\hline$D Q A 1 * 05: 03$ & $38.5 \%$ & $35.3 \%$ & $26.1 \%$ & $21.3 \%$ & $14.4 \%$ \\
\hline \multicolumn{6}{|c|}{ DQB02A Probe } \\
\hline$D Q B 1 * 02: 03$ & $86.1 \%$ & $84.7 \%$ & $82.4 \%$ & $82.7 \%$ & $73.3 \%$ \\
\hline$D Q B 1 * 03: 02$ & $86.3 \%$ & $75.1 \%$ & $65.8 \%$ & $56.2 \%$ & $36.6 \%$ \\
\hline \multicolumn{6}{|c|}{ DQB02gen Probe } \\
\hline$D Q B 1 * 03: 02$ & $44.0 \%$ & $27.0 \%$ & $15.1 \%$ & $7.9 \%$ & $6.5 \%$ \\
\hline \multicolumn{6}{|c|}{ DQB0302A Probe } \\
\hline$\overline{D Q B 1 * 03: 01}$ & $31.5 \%$ & $26.9 \%$ & $14.5 \%$ & $12.2 \%$ & $9.8 \%$ \\
\hline$D Q B 1 * 05: 01$ & $42.3 \%$ & $32.3 \%$ & $15.6 \%$ & $14.2 \%$ & $10.4 \%$ \\
\hline \multicolumn{6}{|c|}{ DQB0302B Probe } \\
\hline$D Q B 1 * 02: 01$ & $69.5 \%$ & $41.3 \%$ & $36.0 \%$ & $34.1 \%$ & $37.4 \%$ \\
\hline$D Q B 1 * 03: 01$ & $67.7 \%$ & $41.3 \%$ & $41.1 \%$ & $36.1 \%$ & $36.1 \%$ \\
\hline \multicolumn{6}{|c|}{ DQA0201 probe } \\
\hline$D Q A 1 * 03: 01$ & $30.3 \%$ & $14.3 \%$ & $3.7 \%$ & $4.1 \%$ & $7.4 \%$ \\
\hline \multicolumn{6}{|c|}{ DQA03 probe } \\
\hline$D Q A 1 * 02: 01$ & $20.3 \%$ & $6.6 \%$ & $4.5 \%$ & $5.5 \%$ & $6.6 \%$ \\
\hline
\end{tabular}

Table 2. 


\begin{tabular}{|c|c|c|c|c|c|}
\hline \multirow[t]{2}{*}{ Probe } & \multicolumn{5}{|c|}{ Sample } \\
\hline & FRCBS 5 & FRCBS12 & FRCBS20 & FRCBS23 & LZL \\
\hline DQA0201 & - & + & - & + & - \\
\hline DQA05 & - & - & + & + & $+/-$ \\
\hline DQA03 & + & + & - & - & - \\
\hline DQB02A & + & + & + & + & - \\
\hline DQB02gen & - & + & + & + & - \\
\hline DQB0302A & + & + & - & - & - \\
\hline DQB0302B & + & + & - & - & - \\
\hline \multicolumn{6}{|c|}{ ELONA Sample Typing } \\
\hline DQ2 & - & - & + & + & - \\
\hline DQ8 & + & + & - & - & - \\
\hline \multicolumn{6}{|c|}{ Alleles present in the sample } \\
\hline \multirow[t]{2}{*}{ DQA region } & 03:01 & 02:01 & 05:01 & 02:01 & 05:03 \\
\hline & 03:01 & 03:01 & 05:01 & 0:501 & $05: 03$ \\
\hline \multirow[t]{2}{*}{ DQB region } & 03:02 & 02:02 & 02:01 & 02:01 & 03:01 \\
\hline & 03:02 & 03:02 & 02:01 & 02:02 & 03:01 \\
\hline \multicolumn{6}{|c|}{ Reference Typing } \\
\hline DQ2 & - & - & + & + & - \\
\hline DQ8 & + & + & - & - & - \\
\hline
\end{tabular}

Table 3: 


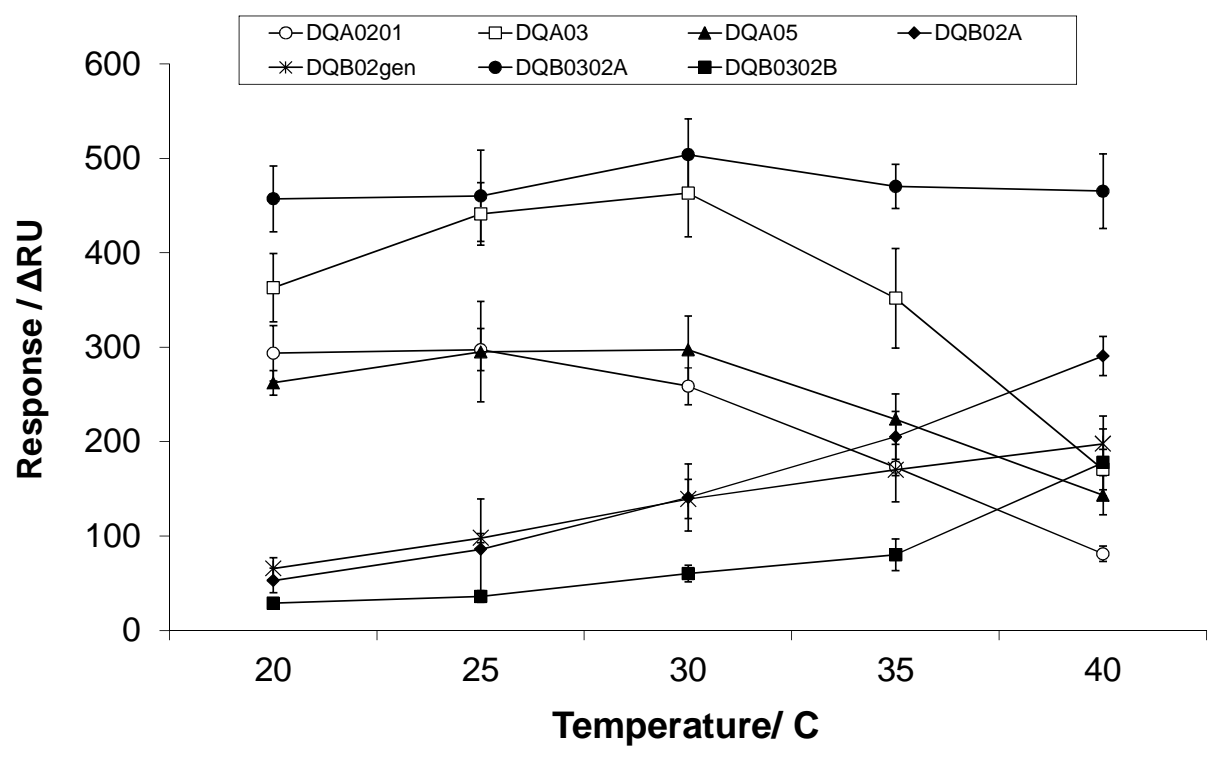

Figure 1 

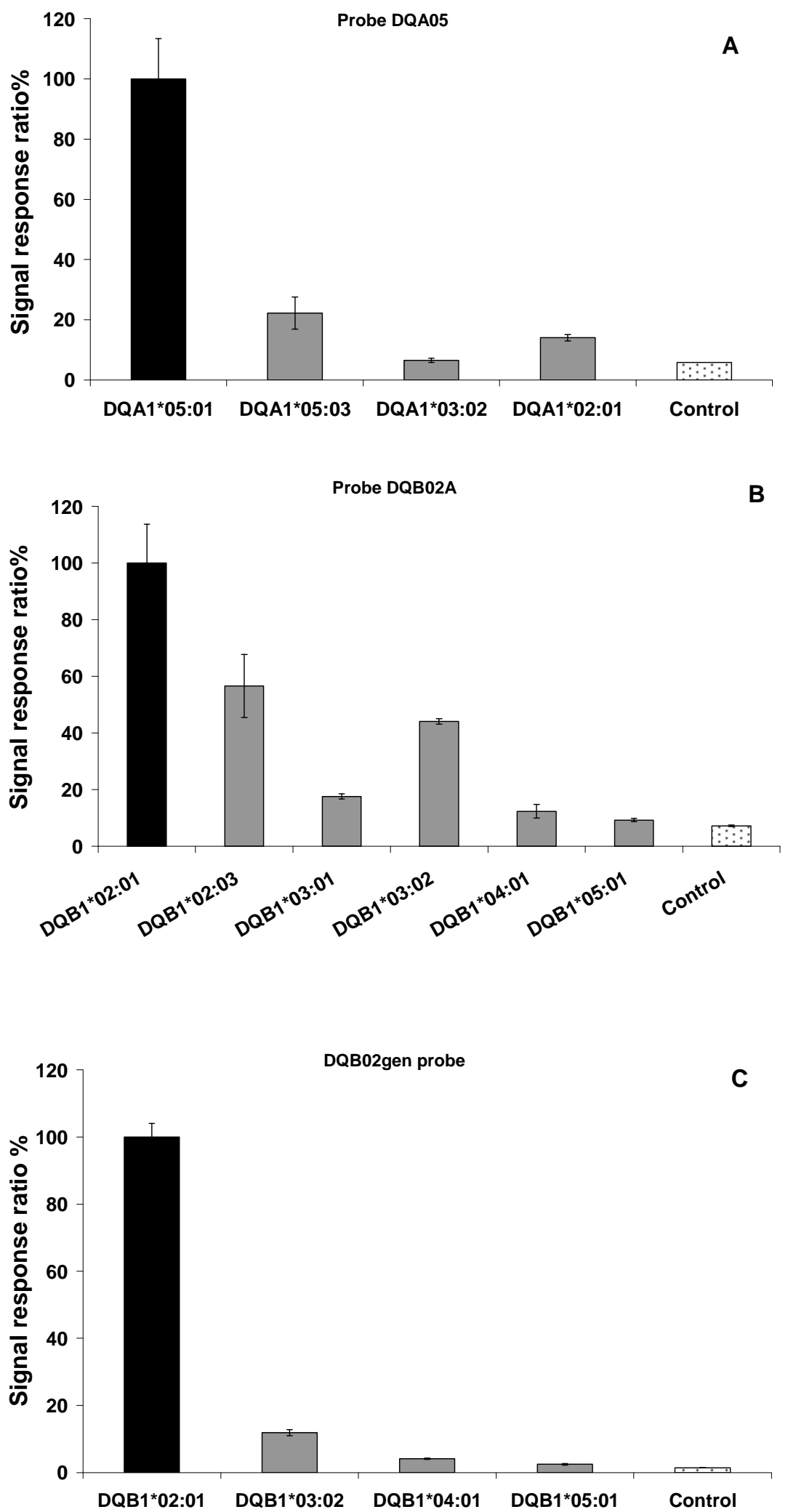

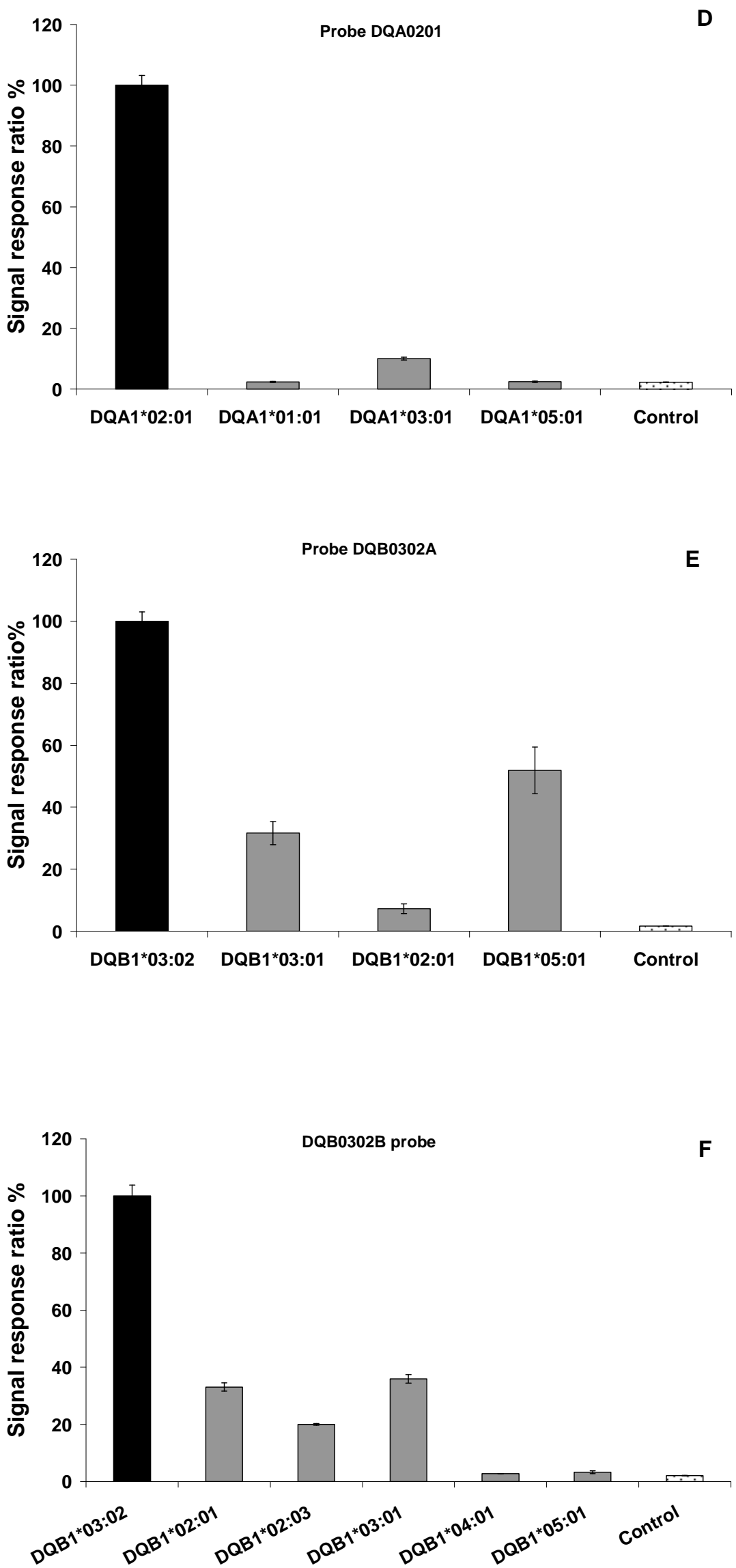


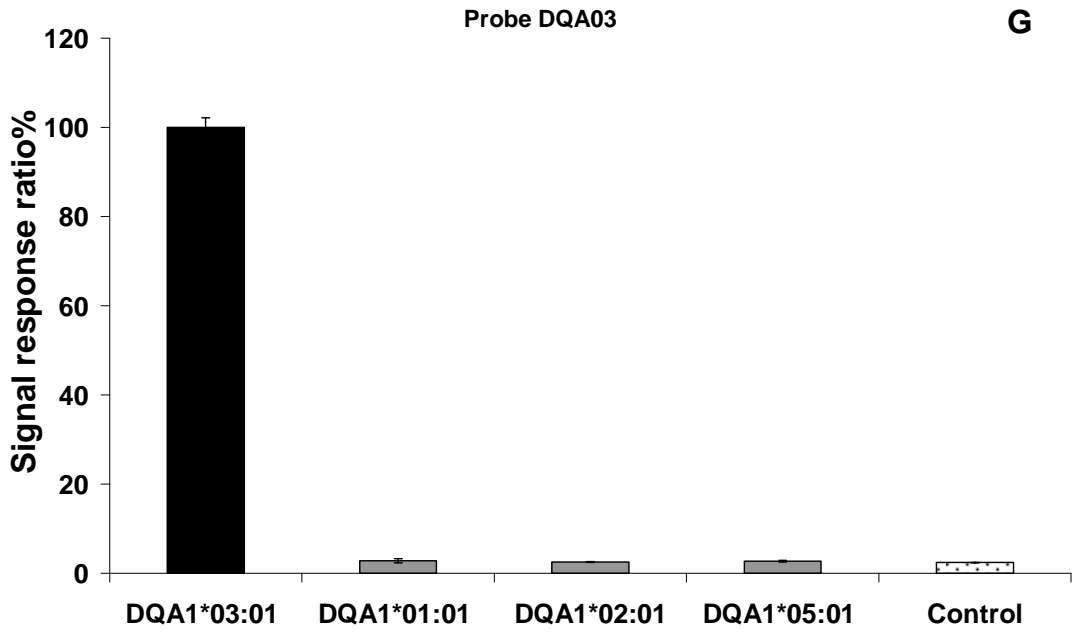

Figure 2 

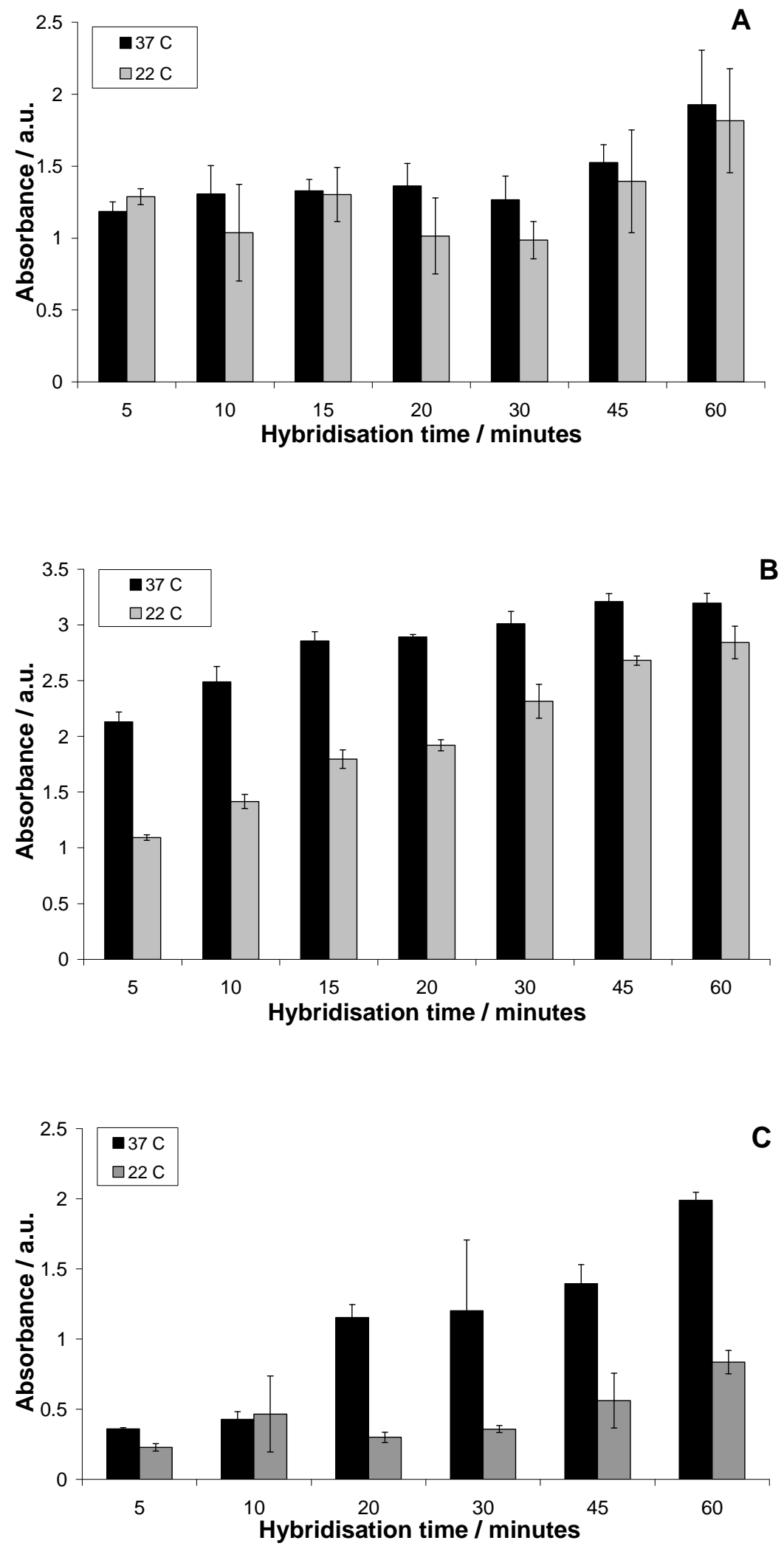


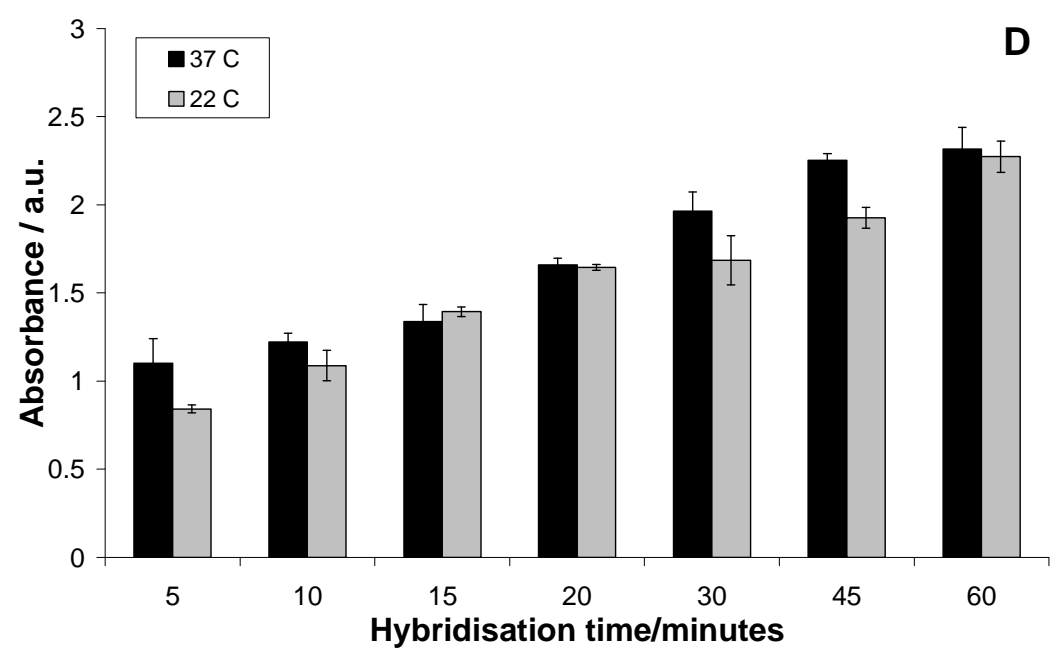

Figure 3 


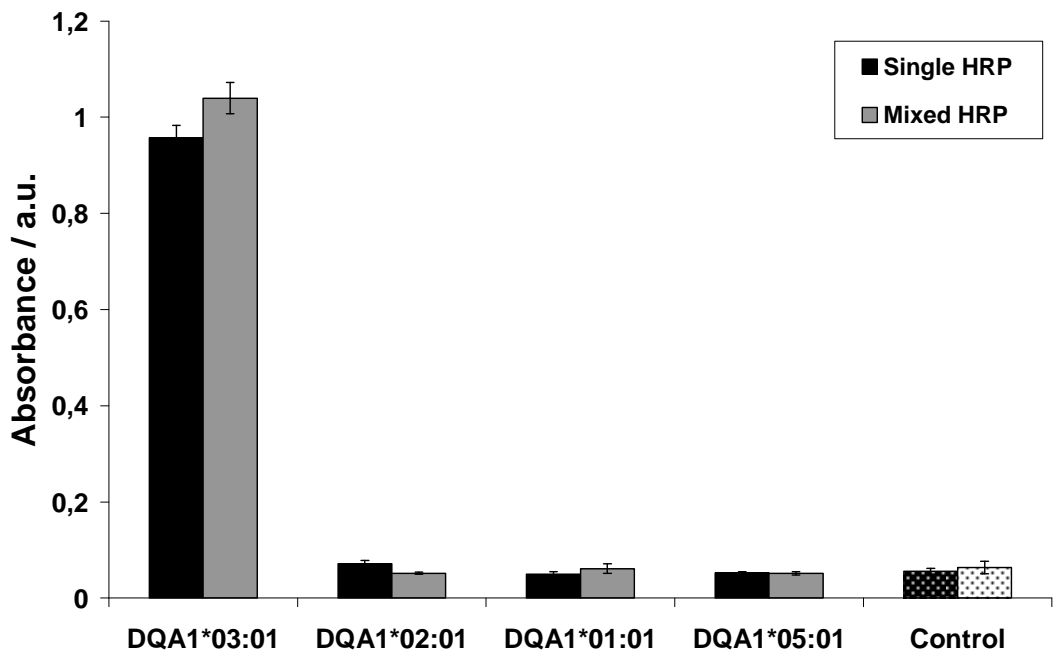

Figure 4: 


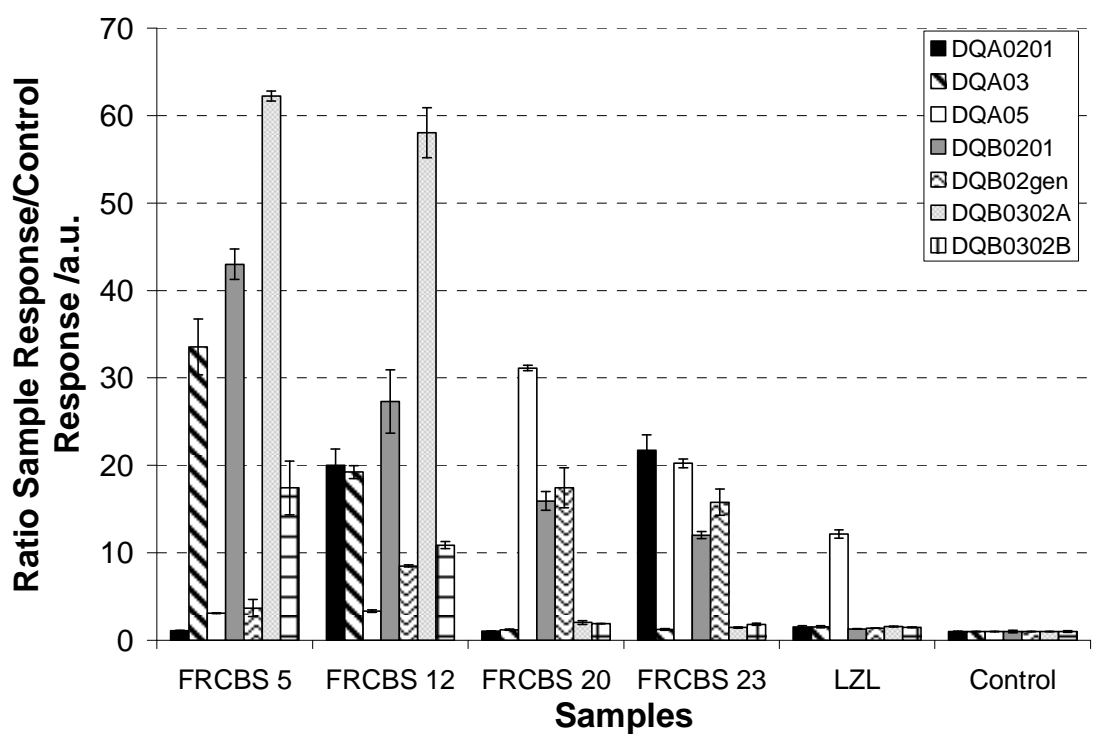

Figure 5 